\title{
A Comparative Study of Some Modifications of CG Methods Under Exact Line Search
}

\author{
Yasir Salih $^{\mathrm{a} *}$, Mustafa Mamat ${ }^{\mathrm{b}}$, and Sukono ${ }^{\mathrm{c}_{*}}$ \\ ${ }^{a}$ Department of Mathematics, Faculty of Education, Red Sea University, SUDAN. \\ ${ }^{a, b}$ Faculty of Informatics and Computing, Universiti Sultan ZainalAbidin, Terengganu, MALAYSIA. \\ ${ }^{c}$ Department of Mathematics, Faculty of Mathematics and Natural Sciences, Universitas Padjadjaran, \\ Bandung, INDONESIA \\ E-mail address: yasirsalih2015@gmail.com; sukono@unpad.ac.id
}

\begin{abstract}
Conjugate Gradient (CG) method is a technique used in solving nonlinear unconstrained optimization problems. In this paper, we analysed the performance of two modifications $\beta_{k}^{Y H M}$ and $\beta_{k}^{M R M}$ and compared the results with the classical conjugate gradient methods of $\beta_{k}^{F R}$ and $\beta_{k}^{P R P}$. These proposed methods possesse global convergence properties for general functions using exact line search. Numerical experiments show that the two modifications are more efficient for the test problems compared to classical CG coefficients.
\end{abstract}

Keywords: Conjugate Gradient Method, unconstrained optimization, exact line search

\section{Introduction}

Consider the following nonlinear unconstrained optimization problems

$$
\min \left\{f(x): x \in R^{n}\right\},
$$

where $f: R^{n} \rightarrow R$ is a continuous differentiable function that is bounded below. To solve (1), starting from an initial point $x_{0} \in R^{n}$, we obtained the next iterative point as follows

$$
x_{k+1}=x_{k}+\alpha_{k} d_{k}, \quad k=0,1,2, \ldots
$$

where $\alpha_{k}$ is the step-length and $d_{k}$ is the search direction defined as;

$$
d_{k}= \begin{cases}-g_{k}, & \text { if } k=0 \\ -g_{k}+\beta_{k} d_{k-1}, & \text { if } k \geq 1\end{cases}
$$


The scalar $\beta_{k}$ is known as the conjugate gradient parameter and the gradient $g_{k}=\nabla f\left(x_{k}\right)$. Some of the classical CG parameters are Fletcher and Reeves (FR) (Fletcher and Reeves, 1964) PolakRibiere-Polyak (PRP) (Polak and Ribiere, 1969; Polyak, 1969) Hestenes-Steifel (HS) (Hestenes and Stiefel,1952) Conjugate Descent (CD) (Fletcher, 1980), Liu-Storey (LS) method (Liu and Storey, 1991). These parameters and are defined as

$$
\begin{aligned}
& \beta_{k}^{F R}=\frac{\left\|g_{k}\right\|^{2}}{\left\|g_{k-1}\right\|^{2}}, \quad \beta_{k}^{P R P}=\frac{g_{k}^{T}\left(g_{k}-g_{k-1}\right)}{\beta_{k-1}^{T} g_{k-1}} \\
& \beta_{k}^{H S}=\frac{g_{k}^{T}\left(g_{k}-g_{k-1}\right)}{d_{k}^{T}\left(g_{k}-g_{k-1}\right)}, \quad \beta_{k}^{C D}=\frac{\left\|g_{k}\right\|^{2}}{d_{k-1}^{T} g_{k-1}} \\
& \beta_{k}^{L S}=\frac{g_{k}^{T}\left(g_{k}-g_{k-1}\right)}{-d_{k-1}^{T} g_{k-1}}, \quad \beta_{k}^{D Y}=\frac{\left\|g_{k}^{2}\right\|}{d_{k-1}^{T}\left(g_{k}-g_{k-1}\right)}
\end{aligned}
$$

Most of these classical methods may not generally be convergent but often possess good convergence property. Recently, more research has been published on new modifications of CG methods. For good references of recent CG methods with significant results, refer to Sulaiman et al. (Sulaiman el al, 2018; Sulaiman et al, 2015; Kamilu et al, 2018; Yasir et al, 2018). Thus, in this paper, we analyszed the performance of two modifications of CG coefficients and compared the perfomance with that of classical CG methods of FR and PRP under exact line search. This is done to improve the overall performance of the resulting algorithms.

\section{Two Modifications and Algorithm}

In this section, we present two modifications known as $\beta_{k}^{Y H M}$ (Saliha et al, 2018) and $\beta_{k}^{M R M}$ (Hamoda et al, 2017) and defined by,

$$
\begin{aligned}
& \beta_{k}^{Y H M}= \begin{cases}\frac{g_{k}^{T}\left(g_{k}-g_{k-1}\right)}{\left\|g_{k-1}\right\|^{2}} \text { if } 0 \leq g_{k}^{T} g_{k-1} \leq\left\|g_{k}\right\|^{2} \\
\frac{g_{k}^{T}\left(g_{k}-\frac{\left\|g_{k}\right\|}{\left\|g_{k-1}\right\|} g_{k-1}\right)}{\left\|g_{k-1}\right\|^{2}} & \text { otherwise }\end{cases} \\
& \beta_{k}^{M R M}=\frac{g_{k}^{T}\left(g_{k}-\frac{\left\|g_{k}\right\|}{\left\|g_{k-1}\right\|} g_{k-1}\right)}{\left\|g_{k-1}\right\|^{2}+\left|g_{k}^{T} d_{k-1}\right|}
\end{aligned}
$$

The following algorithm is a general algorithm for solving unconstrained optimization problems.

\section{Algorithm}

Step1: Given $x_{0} \in R^{n}$, set $\varepsilon>0 d_{0}=-g_{0}$, If $\left\|g_{k}\right\|=0$, then stop. Else,

Step2: Computing step size by,

Step3: Compute

$$
\alpha_{k}=\arg \min _{\alpha \geq 0} f\left(x_{k}+\alpha d_{k}\right) \text {, }
$$

$$
x_{k+1}=x_{k}+\alpha_{k} d_{k} \text {. }
$$


Step4: Compute $\beta_{k}$ and generate $d_{k+1}$ by (3)

Step5: Convergence and stopping criteria. Check if $\left\|g_{k+1}\right\| \leq \epsilon$ stop. Else, Set $k=k+1$ go to Step 2

\section{Global Convergence of the New Modifications}

In this section, we prove the global convergence of $\boldsymbol{\beta}_{\boldsymbol{k}}^{\boldsymbol{Y H} \boldsymbol{M}}$ and $\boldsymbol{\beta}_{\boldsymbol{k}}^{\boldsymbol{M} \boldsymbol{R} \boldsymbol{M}}$ under exact line search. We begin with the sufficient descent condition.

\subsection{Sufficient Descent Condition}

For sufficient descent condition to hold,

$$
g_{k}^{T} d_{k} \leq-c\left\|g_{k}\right\|^{2} \forall k \geq 0 \text { and } c>0 .
$$

The following theorem is used to show our new modification possess with sufficient descent condition under exact line search.

Theorem 1. Let $\left\{x_{k}\right\}$ and $\left\{d_{k}\right\}$ be sequence generated from (2), (3) and the above algorithm, where the step size $\alpha_{k}$ is determined by the exact line search. Then (6) holds for all $\mathrm{k} \geq 0$.

Proof.The prove of this theorem can be found in (Saliha et al, 2018; Hamoda et al, 2017).

\subsection{Global Convergence Properties}

In this section, we prove the global convergence properties of the new modification under some assumptions.

\section{Assumption 1}

(I) $f(x)$ is bounded from below on the level set $\mathrm{R}^{\mathrm{n}}$ and is continuous and differentiable in a neighbourhood $N$ of the set $N=\left\{x \in R^{n}, f(x) \leq f\left(x_{0}\right)\right\}$ at the initial point $\mathrm{x}_{0}$.

(II) The gradient $g(x)$ is Lipschitz continuous in $N$, so there exists a constant $L>0$ such that $\|\mathrm{g}(\mathrm{x})-\mathrm{g}(\mathrm{y})\| \leq \mathrm{L}\|\mathrm{x}-\mathrm{y}\|, \forall \mathrm{x}, \mathrm{y} \in \mathrm{N}$.

The following Lemma by Zoutendijk (Zoutendijk, 1970) is used to prove the global convergence.

\section{Lemma 1.}

Suppose that Assumption 1 holds true. Consider any CG method of the form (3) where $d_{k}$ is the search direction. Then, Zoutendijk condition holds, that is,

$$
\sum_{k=0}^{\infty} \frac{\left(g_{k}^{T} d_{k}\right)^{2}}{\left\|d_{k}\right\|^{2}}<\infty
$$

The following theorem is based on Lemma 1.

\section{Theorem 2}

Suppose assumption 1 holds true, consider $\beta_{k}^{Y H M}$ and $\beta_{k}^{M R M}$ methods of the form (2) and (3) where $\alpha_{k}$ is obtained using exact line search, then, 
$\lim _{k \rightarrow \infty} \inf \left\|g_{k}\right\|=0$

Proof.The prove of this theorem can be found in (Saliha et al, 2018; Hamoda et al, 2017).

\section{Numerical Results}

In this section, we report the detailed numerical results based on the comparisons of our new modifications with classical CG Algorithms of FR and PRP. All algorithms are implemented under exact line search. We selected 31 test functions from Andrei (Andrei, 2008) with different dimensions. We considered $\varepsilon=10^{-6}$ and $\left\|g_{k}\right\| \leq 10^{-6}$ to be the stopping criteria as suggested by Hillstrom (Hillstrom, 1977). In all cases, we used four initial points. These four initial points lead us to test the global convergence of the new modifications. All algorithms are coded on MATLAB Version R2014a. The test was run on an Intel(R) Core ${ }^{\mathrm{TM}}$ i5-M520 $(2.40 \mathrm{GHz}), 4 \mathrm{~GB}$ for RAM memory and Windows 7 Professional operating system. The Numerical results are based on number of iterations and CPU time as presented in Table 1. Also, the performance results of these methods are shown in Figure 1 and Figure 2 respectively, using a performance profile introduced by Dolan and More (Dolan and Moré, 2002).

Table 1: A list of problem functions.

\begin{tabular}{cccc}
\hline No & Function & Dimension & Initial points \\
\hline 1 & Six hump camel & 2 & $-10,-8,8,10$ \\
2 & Booth & 2 & $10,25,50,100$ \\
3 & Treccani & 2 & $5,10,20,50$ \\
4 & Zettl & 2 & $5,10,20,30$ \\
5 & Ex -rosenbrock & $2,4,10,100,500,1000,10000$ & $13,25,30,50$ \\
6 & Extended penalty & $2,4,10,100$ & $50,60,70,80$ \\
7 & Generalized tridiagonal & $2,4,10,100$ & $30,35,40,45$ \\
8 & Shalow & $2,4,10,100,500,1000,10000$ & $10,25,50,70$ \\
9 & Ex - tridiagonall & $2,4,10,100,500,1000,10000$ & $12,17,20,30$ \\
10 & Extended white and holst & $2,4,10,100,500,1000,10000$ & $3,10,30,50$ \\
11 & Quadrtic QF2 & $2,4,10,100,500,1000$ & $10,30,50,100$ \\
12 & Extended denschnb & $2,4,10,100,500,1000,10000$ & $8,13,30,50$
\end{tabular}


13

14

15

16

17

18

19

20

21

22

23

24

25

26

27

28

29

30

31
Hager

Ex-powell

Extended beale

Ex -himmelblau

Diagonal 2

Perturbed quadratic

Sum Squares function

Ex- quadratic penalty QP2

Raydan1 function

Generalized tridiagonal 2

Quadratic QF1

Dixon and Price

Fletcher

Ex- maratos

Leon function

Extended wood

Quartic function

Matyas function

Colville function
$2,4,10,100$

$4,8,20,100,500,1000$

$2,4,10,100,500,1000,10000$

$100,500,1000,10000$

$2,4,10,100,500,1000$

$2,4,10,100,500,1000$

$2,4,10,100,500,1000$

$4,10,100,500,1000,10000$

$2,4,10,100$

$2,4,10,100$

$2,4,10,100,500,1000$

$2,4,10,100$

$4,10,100,500,1000$

$2,4,10,100$

2

4

4

2

4
$1,3,5,7$

$-1,1,7,11$

$-19,1,13,23$

$50,70,100,125$

$-1,1,2,3$

$1,5,10,15$

$1,10,20,30$

$17,18,19,20$

$1,3,5,7$

$1,7,8,14$

$1,2,3,4$

$100,125,150,175$

$7,11,13,15$

$5,10,12,15$

$2,5,8,10$

$3,5,20,30$

$5,10,15,20$

$5,10,15,20$

$2,4,7,10$

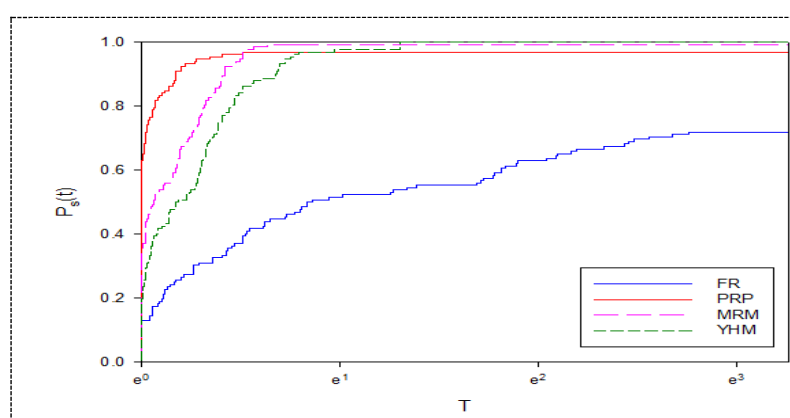

Figure 1: Performance profile based on number of iteration.

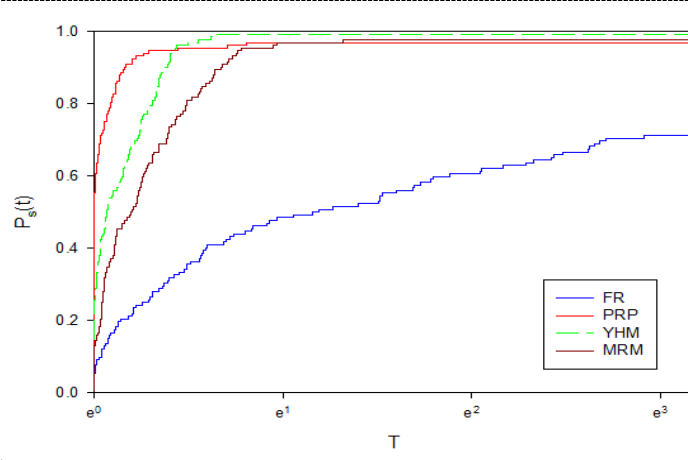

Figure 2. Performance profile based on CPU Time. 
From the above figures, it can be seen that the new modifications are better than the classical methods of FR and PRP based on number of iteration and CPU times. The results show that the proposed method solves $99.4 \%$ and $98 \%$ of the test problems respectively. Meanwhile, FR method solves about $72 \%$ while PRP solves $97 \%$ of the test problems. Hence, we can say that $\beta_{k}^{Y H M}$ and $\beta_{k}^{M R M}$ are more efficient with robust performance.

\section{Conclusion}

In this paper, we presented the performance of two new modifications for nonlinear unconstrained optimization and compared them with the classical CG coefficient of FR and PRP methods under exact line search. These proposed methods possess the global convergence condition under some assumptions and satisfy the sufficient descent condition. Numerical results showed that the proposed methods outperformed the classical methods of FR and PRP in solving the standard unconstrained optimization problems.

\section{Acknowledgements}

The authors would like to thank Universiti Sultan Zainal Abidin (UniSZA) and Red Sea University of Sudan for financial suport.

\section{References}

Andrei N. (2008). An unconstrained optimization test functions collection. Adv. Model. Optim, 10(1): p. 147-161.

Dolan E.D and Moré JJ. (2002). Benchmarking optimization software with performance profiles. Mathematical programming, 91(2): p. 201-213.

Fletcher R and Reeves C M. (1964). Function minimization by conjugate gradients. The computer journal, 7(2): p. 149-154.

Fletcher R. 1980. Practical methods of optimization. Vol. 1, Unconstrained Optimization.

Hamoda M, Rivaie M and Mamat M. (2017). A New Nonlinear Conjugate Gradient Method With Exact Line Search For Unconstrained Optimization. Journal of Humanities and Applied Science (JHAS), pp. 1-16.

Hestenes MR and Stiefel E. (1952). Methods of conjugate gradients for solving linear systems. Vol. 49: NBS.

Hillstrom K.E. (1977). A simulation test approach to the evaluation of nonlinear optimization algorithms. ACM Transactions on Mathematical Software (TOMS), 3(4): p. 305-315. 1977. 3(4): p. 305-315. 
Kamilu, K., Sulaiman' I. M., Waziri' M. Y., and Abashar, A. (2018). Another Improved Three Term PRP-CG Method with Global Convergent Properties for Solving Unconstrained Optimization Problems. Malaysian Journal of Computing and Applied Mathematics. 1(1), $1-10$.

Liu Y and Storey C. 1991.Efficient generalized conjugate gradient algorithms, part 1: theory. Journal of optimization theory and applications, 69(1): p. 129-137.

Polyak B.T. (1969). The conjugate gradient method in extremal problems. USSR Computational Mathematics and Mathematical Physics, 9(4): p. 94-112

Polak E and Ribiere G. (1969). Note sur la convergence de méthodes de directions conjuguées. Revue française d'informatique et de recherche opérationnelle. Série rouge, 3(16): p. 3543.

Saliha, Y., M.A. Hamoda, and M. Rivaiec. (2018). New Hybrid Conjugate Gradient Method with Global Convergence Properties for Unconstrained Optimization. Malaysian Journal of Computing and Applied Mathematics, 1(1): p. 29-38

Sulaiman, I. M., Mamat, M., Abashar, A., and Zabidin, S. (2015). A Modified Nonlinear Conjugate Gradient Method for Unconstrained Optimization, Applied Mathematical Sciences 9(54), $2671-2682$.

Sulaiman' I. M., Waziri' M. Y., Olowo' E. S., and Talat' A. N. (2018). Solving Fuzzy Nonlinear Equations with a New Class of Conjugate Gradient Method. Malaysian Journal of Computing and Applied Mathematics. 1(1), 11-19.

Yasir, S., Mohamed, A. H., and Rivaie, M. (2018). New Hybrid Conjugate Gradient Method with Global Convergent Properties for Solving Unconstrained Optimization. Malaysian Journal of Computing and Applied Mathematics. 1(1), 29-38.

Zoutendijk G. (1970). Nonlinear programming, computational methods. Integer and nonlinear programming, 143(1): p. 37-8 